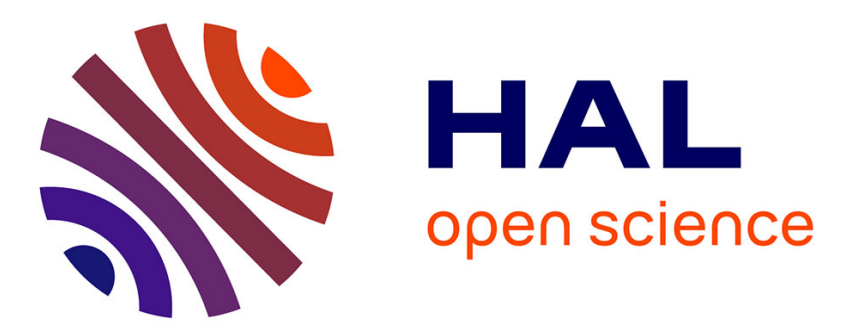

\title{
Thermoluminescence study of the trapped charge at an alumina surface electrode in different dielectric barrier discharge regimes
}

P F Ambrico, M Ambrico, A Colaianni, L Schiavulli, G Dilecce, S de Benedictis

\section{To cite this version:}

P F Ambrico, M Ambrico, A Colaianni, L Schiavulli, G Dilecce, et al.. Thermoluminescence study of the trapped charge at an alumina surface electrode in different dielectric barrier discharge regimes. Journal of Physics D: Applied Physics, 2010, 43 (32), pp.325201. 10.1088/0022-3727/43/32/325201. hal-00569669

\section{HAL Id: hal-00569669 \\ https://hal.science/hal-00569669}

Submitted on 25 Feb 2011

HAL is a multi-disciplinary open access archive for the deposit and dissemination of scientific research documents, whether they are published or not. The documents may come from teaching and research institutions in France or abroad, or from public or private research centers.
L'archive ouverte pluridisciplinaire HAL, est destinée au dépôt et à la diffusion de documents scientifiques de niveau recherche, publiés ou non, émanant des établissements d'enseignement et de recherche français ou étrangers, des laboratoires publics ou privés. 


\title{
1 Thermoluminescence study of the trapped charge at alumina 2 surface electrode in different dielectric barrier discharges 3 regimes.
}

\author{
P F Ambrico $^{1}$, M Ambrico ${ }^{1}$, A Colaianni ${ }^{2,4}$, L Schiavulli ${ }^{3,4}$, G Dilecce ${ }^{1}$ and S De Benedictis ${ }^{1}$. \\ 1 Consiglio Nazionale delle Ricerche, Istituto di Metodologie Inorganiche e dei Plasmi UOS Bari - clo Dipartimento di \\ Chimica, via Orabona, 4, 70126 Bari, Italy \\ 2 Dipartimento di Geologia e Geofisica - via Orabona, 4, 70126 Bari, Italy \\ 3 Dipartimento Interateneo di Fisica, - via Orabona, 4, 70126 Bari, Italy \\ 4 INFN sezione di Bari, via Orabona, 4, 70126 Bari, Italy \\ PACS 52.25.-b \{Plasma properties \\ PACS 52.25.Mq \{Dielectric properties\} \\ PACS 51.50.+v \{Electrical properties\} \\ PACS 78.60.Kn \{Thermoluminescence\} \\ PACS 72.20.Jv \{Charge carriers: generation, recombination, lifetime, and trapping\}
}

E-mail: paolofrancesco.ambrico@cnr.it

\begin{abstract}
In the present study charge trapping effect in alumina dielectric surfaces has been deeply investigated by means of a dedicated dielectric barrier discharge apparatus under different discharge regime and gas mixtures. These work further validates our previous findings in the case of air discharges under filamentary regime. The long lasting charge trapping has been evidenced by ex-situ thermoluminescence characterizations of alumina dielectric barrier plates exposed to plasma. The density of trapped surface charges was found to be higher in the glow discharge with respect to pseudoglow and filamentary regimes and for all regimes a minimum trap activation temperature was of $390 \mathrm{~K}$ and trap energy less than or around $1 \mathrm{eV}$. This implies that in the case of glow discharges a higher reservoir of electrons is present. Also, the effect was found to persist for several days after running the discharge.
\end{abstract}

\section{Introduction}

The surface charge trapping at dielectric surface due to exposure to atmospheric pressure dielectric barrier discharge (DBD) plasma can influence the discharge regime [1]; in fact surface charges act as a reservoir that can contribute to stabilize a glow discharge regime for instance in air discharges [2]. Golubovskii et al. suggested that many seed electrons necessary for a uniform/homogeneous discharge could be produced by electrons trapped on the dielectric surface [3]. Recently, long lifetime of charge trapping on alumina used as dielectric in DBD plasma has been evidenced by Thermally stimulated current [1] and Thermoluminescence technique [4]. We found that the adsorbed electrons in the case of alumina were trapped in shallow traps during plasma running and have mainly an energy of about $1 \mathrm{eV}$ [4], i.e. at a much lower energy than the intrinsic alumina electrons and much easier to be removed from the dielectric. These trapped electron could 
also explain the fact that irradiating the dielectric surface with low fluence laser beam could result in the triggering of a discharge for a voltage lower than the breakdown value [5].

From what stated above, it follows that different discharge regimes are expected to act differently on the surface charge trapping since the plasma electron energy distribution and current can be different. It is also furthermore known that DBD can run under filamentary or diffuse mode depending on discharge geometry and gas mixture. For instance, the nitrogen discharge can be run in different regime depending on the geometry and other discharge parameters[6,7]. Moreover, the addiction of small quantities of oxygen to a nitrogen discharge transform the regime from glow to filamentary [8]. Conversely, running an helium discharge this will lead to a quite distinct glow regime [9].

The aim of this work is to study the surface charge trapping effect under different discharge regime and gas mixture at atmospheric pressure running in a dedicated experimental set up. The effect of surface charge trapping on the dielectric barrier plates after plasma exposure will be evidenced by ex-situ Thermo Luminescence glow curve (TL GC) measurements as already preliminarly described in ref.4. The correlation between the discharge regimes and and the observed glow curves parameters will be discussed.

\section{Experimental}

A DBD discharge apparatus has been build up in order to easily control the surface area exposed to the plasma; respect to previous experimental configuration [4], each single specimen of alumina (mean diameter $0.5 \mathrm{~mm}$ ), constitute one of two dielectric barrier plates, covering the metallic electrodes, exposed to plasma. A drawing of the discharge apparatus is shown in figure 1.

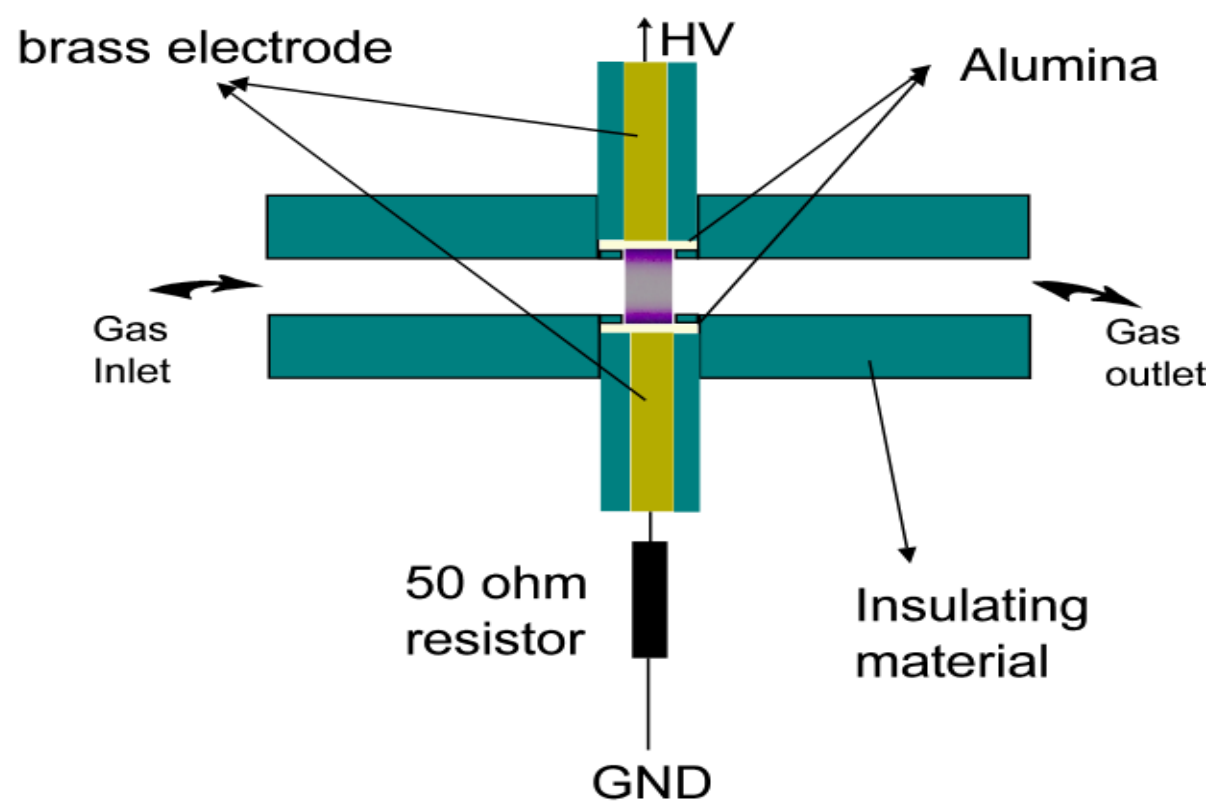

Figure 1. Schematic of the experimental apparatus (colour online).

The parallel plates discharge is composed of two brass electrode $3 \mathrm{~mm}$ in diameter, two $0.7 \mathrm{~mm}$ thick alumina plates (Coorstek fine grain, 96\% purity) that completely cover the electrodes and two spacers designed to vary the discharge gap from a minimum of $2 \mathrm{~mm}$ (for $\mathrm{N}_{2}$ main gas mixtures) and $5 \mathrm{~mm}$ for $\mathrm{He}$ 
main gas mixtures. The minimum distance between electrodes is limited by the geometry of the electrode support. The electrodes are completely isolated by highly insulating plastics except the surface in contact with the aluminum dielectrics. The discharge vessel is made of a stainless steel and PVC flanges for the high voltage (HV) connections and is equipped with vacuum fittings.

Figure 2. Typical voltage and current waveforms for: (A) $\mathrm{He}$, (B) $\mathrm{He}-50 \% \mathrm{~N}_{2},(C) \mathrm{N}_{2}$, (D) $\mathrm{N}_{2}-20 \% \mathrm{O}_{2}$, (E) $\mathrm{O}_{2}$ plasmas.
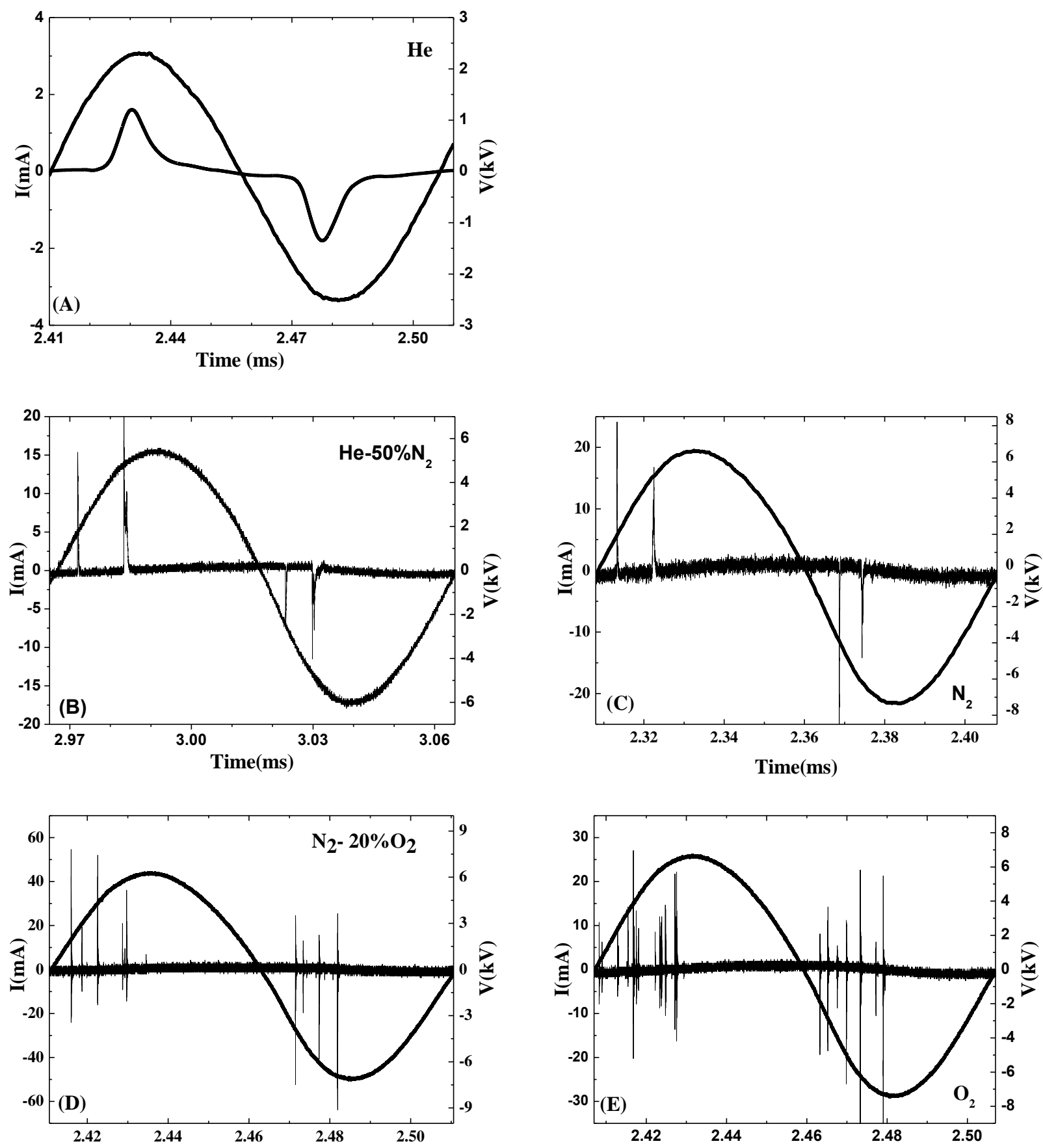

Time(ms)

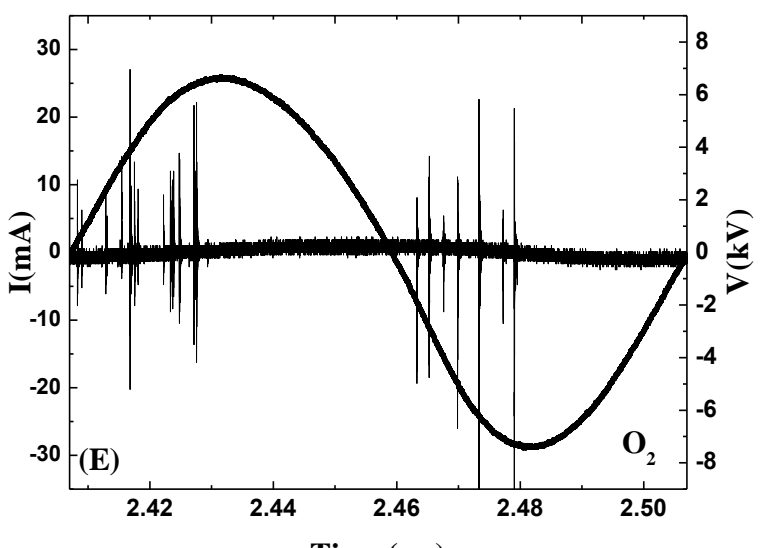

Before operation the discharge volume is evacuated to few tens of mTorr by a $30 \mathrm{~m}^{3} / \mathrm{h}$ rotary pump. The gas flow is operated by MKS flow meters/controllers, and pumping through an adjustable needle valve is used to maintaining the 760 Torr pressure under a total gas flow of $1000 \mathrm{sccm}$. Pure $\mathrm{N}_{2}, \mathrm{O}_{2}$ and $\mathrm{He}$ (impurities less than $6 \mathrm{ppm}$ ) are used as gases supply. The HV supply is composed of a low voltage sinusoidal generator 
(Wavetek mod. 164), a power amplifier (Industrial Test Equipment Powerton 1000A), and a high voltage transformer. The applied voltage is pulse modulated with duty cycle $\mathrm{T}_{\text {on }}=\mathrm{T}_{\text {off }}=5 \mathrm{~ms}$. Different discharge regimes have been produced under different plasma mixture. Specifically, a filamentary regime will be typical of $\mathrm{N}_{2}-20 \% \mathrm{O}_{2}$ (air like mixture) and $\mathrm{O}_{2}$ discharge, a diffuse range will characterize that of He while an intermediate regime will be that of $\mathrm{N}_{2}$ and He- $50 \% \mathrm{~N}_{2}$ discharges. The discharge parameter, current and voltage, have been continuously monitored and registered during each run. Typical oscillograms of the applied voltage and discharge current for DBD for a total pressure of 760 torr, flow rate of 1 liter/minute and different gas mixture area reported in figure 2.

The discharge regimes characterizing the working conditions of DBDs in the present study are shown in figure 2. In the case of Helium discharges, the DBD is homogeneous/diffuse along the plane of the electrode and in the discharge gap. The glow character is associated to one major current peak for half cycle. The discharge in nitrogen and nitrogen helium is homogeneous and assume the pseudoglow [10] discharge aspect, with usually a first current pulse which has a faster rise time and shorter duration, and a second pulse in both polarities which has not only an appreciably longer rise time but also a rather long decay time constant. Both current peak have a pulse duration of few microseconds. In the case of air and oxygen the discharge is in a filamentary state that is consisting of a large number of narrow filaments stochastically distributed over the electrode area. The discharge current has the form of multiple peaks (micro-discharges) of some tens of nanosecond duration.

From the current measurements the electron current transferred charge can be estimated by the integration of the current peak, using $\mathrm{Q}=\int \mathrm{i}(\mathrm{t}) \mathrm{dt}$ for a current peak recorded in single-shot after subtraction of the displacement current. Of course the charge measured using this method is generally slightly underestimated [11] in the case of filamentary regime, in which faster signal are involved and the oscilloscope sampling can be affected by higher error. Since the current for one cycle is monitored continuously an average transferred charge per cycle can be easily estimated, and the total current transferred charge can be estimated by multiplying it for the total number of cycles during the measurements period.

107 During all the experiments several alumina disk plates $(\varnothing=6 \mathrm{~mm}$ and $0.7 \mathrm{~mm}$ thick) taken from the same $1087 \times 7 \mathrm{~cm}^{2}$ slabs were used as dielectric barrier of ground electrode so to be easily removed after discharge run 109 and analysed ex-situ by TL apparatus.

110 The thermoluminescence glow curves were collected on alumina samples gas plasma by using an automated 111 RIS $\varnothing$ TL/OSL-DA-15 [12] reader equipped with ${ }^{90} \mathrm{Sr} /{ }^{90} \mathrm{Y}$ beta $(\beta)$ source radiation $(0.565 \mathrm{MeV}$ mean $\beta$ 112 energy, dose rate $0.119 \mathrm{~Gy} / \mathrm{s}$ ). The system is equipped with a photomultiplier tube (bi-alkali EMI 9235QA), 113 which has a maximum detection efficiency at approximately $400 \mathrm{~nm}$, at a distance of $55 \mathrm{~mm}$ from the 114 sample. The spectral selection was accomplished by means of a $7.5 \mathrm{~mm}$ Hoya U-340 detection filter $\left(\lambda_{\text {peak }}=\right.$ $115340 \mathrm{~nm}, \mathrm{FWHM}=80 \mathrm{~nm}$ ).

116 The thermoluminescence glow curves have been acquired by heating the sample in an inert nitrogen 117 atmosphere (2 liter/minutes flow) at a heating rate of $5 \mathrm{~K} / \mathrm{s}$ in the temperature range $323 \mathrm{~K}-673 \mathrm{~K}$. The 118 measurement is accomplished in 90 seconds. 
119 All the alumina samples were preliminarily classified (after thermal annealing at $800^{\circ} \mathrm{C}$ for two hours) by 120 means of Thermoluminescence measurements, that was used as reference for the sample, following a typical 121 radiation dosimetry protocol:

122 a) thermoluminescence background;

123 b) 90 s beta irradiation;

124 c) thermoluminescence glow curve;

125 Before each plasma exposure the alumina disks were thermally cleaned at $800^{\circ} \mathrm{C}$ for two hours, in order to 126 deplete spurious trapped charges due to exposure to environmental radiation.

127 After plasma exposure the alumina disks have been promptly dismounted in dark conditions stored in a black 128 box, to avoid or reduce any possible thermo luminescence glow curve intensity reduction due to bleaching 129 effect, in dry air and mounted in the TL apparatus. The overall time from the switch off of the plasma to the 130 start of the Thermoluminescence measurements was about 20 minutes. The Plasma induced luminescence 131 [13] counts at the beginning of the measurements in the UV region covered by the TL filters were negligible. 132 The Thermoluminescence protocol after plasma exposure was the following:

133 a) Thermoluminescence readout (that takes about 90 seconds);

134 b) Thermoluminescence background. In all the sample after different plasma exposure the collected 135 background was comparable to the reference indicating that no luminescence was coming from the sample 136 after the thermoluminescence signal readout. We can exclude thermally activated reaction from the surface 137 as reported in [13].

138 c) Control Thermoluminescence readout following the beta radiation exposure protocol. For all the samples 139 the beta irradiated thermoluminescence were always very close to the control one collected at the beginning. 140 This is an indication that the surface was not modified by the plasma or for beta radiation damage or 141 sensitization.

142 Plasma exposure times have been varied ranging from 5 to 90 minutes in the case of air discharge and from 5 143 to 30 minutes in the case of $\mathrm{He}$ and $\mathrm{He}_{2}$ discharges. In the case of $\mathrm{He}$ two different high voltage values (3 $144 \mathrm{kV}_{\mathrm{pp}}$ and $5 \mathrm{kV}_{\mathrm{pp}}$ ) were used that is leading to different discharge current. As a matter of comparison, 145 thermoluminescence glow curves were also collected on a set of alumina samples after $\beta$ exposure and 146 varying the irradiation time from 60 s to 2100 s i.e. for a total dose ranging from $7 \mathrm{~Gy}$ to $250 \mathrm{~Gy}$.

147 The thermoluminescence glow curves have been analyzed by using the free downloadable GLOW FIT 148 program [14] capable of simultaneously processing up to ten TL glow peaks following the first order kinetics 149 model [15].

\section{Results and discussion}

\subsection{The thermoluminescence technique}

153 The charge trapping effect due to plasma exposure was approached by using the thermoluminescence 154 properties of the dielectric plates used as barrier in DBD discharge. It could be useful to remind that in a pure 155 insulator (but this hold also for semiconductors) there are two relevant energy bands: (i) an almost 
completely filled valence band and (ii) an almost empty conduction band separated by a forbidden gap (called band-gap energy $\mathrm{E}_{\mathrm{g}}$ ). Transitions of electrons between the valence band and the conduction band can occur if the valence band electron acquires an energy $\geq \mathrm{E}_{\mathrm{g}}$.

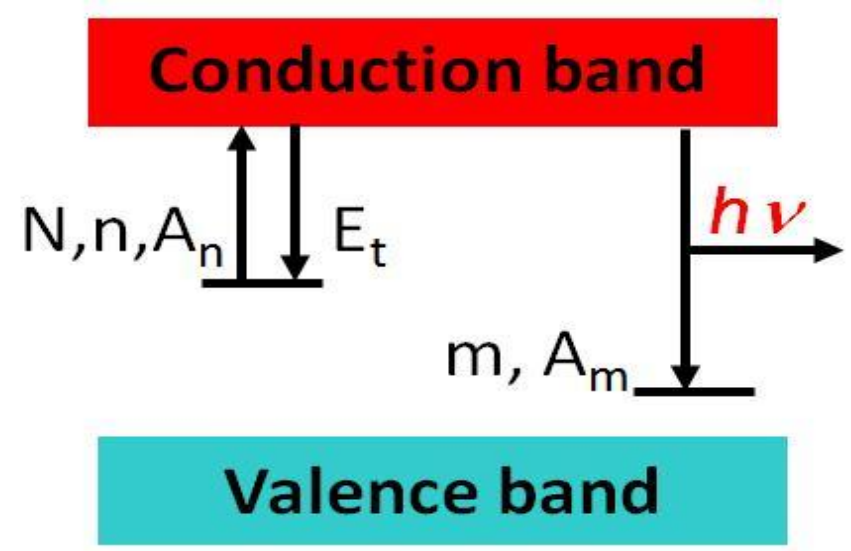

159

160

Figure 3. Scheme of principle of charge trapping/detrapping and recombination mechanisms in an insulating material leading to a luminescence (or thermoluminescence) effect. Here the simplest case on one trap and one recombination center energy distribution are displayed. $\mathrm{N}$ is total trap state concentration, $\mathrm{n}$ the concentration of trapped electrons; $\mathrm{m}$ is concentration of available hole states (for recombination); $E_{t}$ is the trap energy; $A_{n}$ is the retrapping probability and $A_{m}$ is the recombination probability (colour online).

Imperfections in the crystal, associated with impurities and/or lattice defects may create new localized energy levels in the forbidden band gap. Under certain conditions, some of these defects are capable to trap an electron. One of this conditions is ionizing radiation exposure producing free electrons that can be trapped on one of more than of these sites. Some of these traps can be thermally depleted and recombine on recombination centers emitting a photon. This is the basic phenomenon on which the thermoluminescence technique works. The trap determined after a TL measurements is characterized by the energy $\mathrm{E}_{\mathrm{t}}$ (which is referred to as the trap depth) that an electron must acquire from lattice vibrations to escape to the conduction band where it can move freely in the crystal and by the characteristic temperature $\mathrm{T}_{\mathrm{m}}$ at which the thermal vibrations of the crystal lattice are sufficient to cause the release of trapped electrons. The rate at which the electrons, or holes, escape from the traps is roughly governed by the vibrational frequency (or attempt-toescape factor or frequency) $s$ of the charge within the trap and the trap depth E. The overall escape rate is proportional to $S \cdot \exp \left(-E_{\ell} / k T\right)$.

The thermally stimulated light emission from an insulator or a semiconductor following the previous absorption of energy from ionizing radiation results in the typical Thermoluminescence glow curve from which the information on the electron traps present in the band gap can be extracted. By looking at Figure 3, under the hypothesis that the total electron retrapping probability $(N-n) A_{n}$ is much lower that the recombination probability $m A_{m}$ (i.e. slow retrapping condition), the thermoluminescence intensity for single peak is given by the well known first order kinetic equation [16] in the case of a linear temperature gradient: 


$$
I(T)=n_{0} S \exp \left(-\frac{E_{t}}{k T}\right) \exp \left(-\frac{S}{\delta} \int_{T_{0}}^{T} \exp \left(-\frac{E_{t}}{k T^{\prime}}\right) d T^{\prime}\right)
$$

Where $\delta$ is the heating rate, $\mathrm{T}_{0}$ is the initial temperature, $\mathrm{n}_{0}$ the initial number of filled traps, $\mathrm{E}_{\mathrm{t}}$ is the trap energy, $S$ is the above expressed frequency factor $\left(\mathrm{s}^{-1}\right)$ indicating also the number per second an electron interacts with the lattice. The exponential integral in equation (1) could be only analytically solved [17] and gives a representation of the single peak glow curve as a non linear function of trap energy $\mathrm{E}_{\mathrm{t}}$, peak temperature at maximum $\mathrm{T}_{\mathrm{M}}$ and peak maximum intensity $\mathrm{I}_{\mathrm{M}}$. The integral in (1) is then the representation of a glow peak with a maximum intensity $\mathrm{I}_{\mathrm{M}}$ at a characteristic temperature $\mathrm{T}_{\mathrm{M}}$ following the equation:

$$
\frac{\delta E_{t}}{k T_{M}^{2}}=S \exp \left(-\frac{E_{t}}{k T_{M}}\right)
$$

It then follows that the integral light intensity depends on the number of trapped charges that are in turn depending on the irradiation dose and independent on the heating cycle. The characteristic temperature $T_{M}$ does not depend on the number of trapped charges and relates with the trap energy $E_{t}$. Moreover, the trap energy depth $E_{t}$, the peak maximum temperature $T_{M}$ and the width of the peak $\omega$ (defined as the ratio $\mathrm{A} / \mathrm{I}_{\mathrm{M}}$ i.e. between the glow peak area and the peak intensity at its maximum), are related among them by the following relation [18]:

From eq.(3) it should be expected that glow peak at higher temperature correspond to deeper traps. However, when complex glow curve resulting from peak overlapping are analyzed this is not a followed rule since also the width of glow peak contributes in defining the correspondence between the energy and glow peak maximum temperature[18]

The analysis of such complex curves can be nowadays afforded by a friendly free downloadable software namely GlowFit program [14] specially designed to simulate thermoluminescence glow curve resulting from up ten single or superimposed glow peaks following the first order kinetics. The GlowFit software allows to determine the trap energy depth $E_{t}$, the peak maximum temperature $T_{M}$, the peak maximum intensity $I_{M}$ and the frequency factor $S$ as output best fit parameters and calculates the peak area A. Moreover, the goodness of the fit is expressed through the figure of merit (FOM) value that is a further program output and is defined as :

$$
\operatorname{FOM}(\%)=\sum_{i=1}^{n} 100 \cdot \frac{\left|\Delta y_{i}\right|}{A_{T}}
$$

where $n$ is the number of data points, $\Delta \mathrm{y}_{\mathrm{i}}$ is the difference between the experimental and fitted points and $\mathrm{A}_{\mathrm{T}}$ is the integral of the fitted glow curve in the region of interest [19].

\subsection{Effect of different discharge regimes}

218 In figure 3 the collected thermoluminescence glow curves (TL GCs) for a 30 minutes plasma exposure time 
the terminology used for the $\beta$ radiation, we will refer to "plasma radiation" as the sum of all agents 221 (electrons, photons, ions) that, impinging on the dielectric,are capable to excite an electron into a surface trap level and term "plasma radiation dose" the corresponding estimated dose. The TL GC intensity of the different observed spectra shown in figure 4 relates well with the measured current in the different plasma regime conditions. In fact the higher electron current transferred charge is obtained in the helium plasma glow regime, then the pseudo-glow regime of helium-nitrogen and nitrogen plasma and last the filamentary regime of air and oxygen plasma (see table 1).

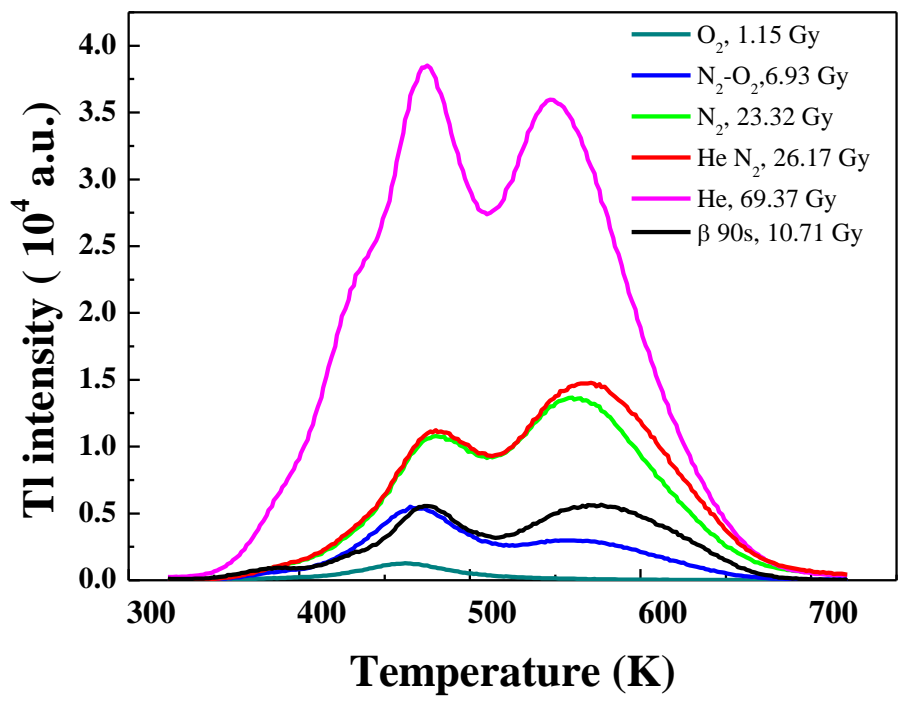

Figure 4. Thermoluminescence glow curves after alumina exposed to different gas mixture discharge plasma. The GC data refers to 30 minutes plasma exposure. Glow curve after $90 \mathrm{~s}$ of $\beta$ exposure is also shown for comparison. (colour online)

It is evident that exposing the alumina samples for the same time to different plasma produces thermoluminescence glow curves with different intensities. Furthermore, the plasma induced thermoluminescence glow curves display features similar to those observed after $\beta$ irradiation which is constituted of high energetic electron current. Possible effects of the alumina exposure to UV radiation although present in the plasma as irradiation source have been excluded since, as previously observed, glow curve observed after UV (mercury lamp) exposure display a different shape due to the UV light induce bleaching effect [4]. Respect to previously published results for the case of synthetic air [4], referring to glow curve collected after some days from plasma exposure, the short delay between the $\mathrm{N}_{2}-\mathrm{O}_{2}$ plasma exposure and thermoluminescence measurements results in a much more intense glow curve.

241 The radiation dose after plasma exposure was estimated by using the known $\beta$ radiation source dose rate as a reference. First of all the linear dependence of the thermoluminescence glow curve area on the total $\beta$ radiation dose has been verified (see figure 5) 


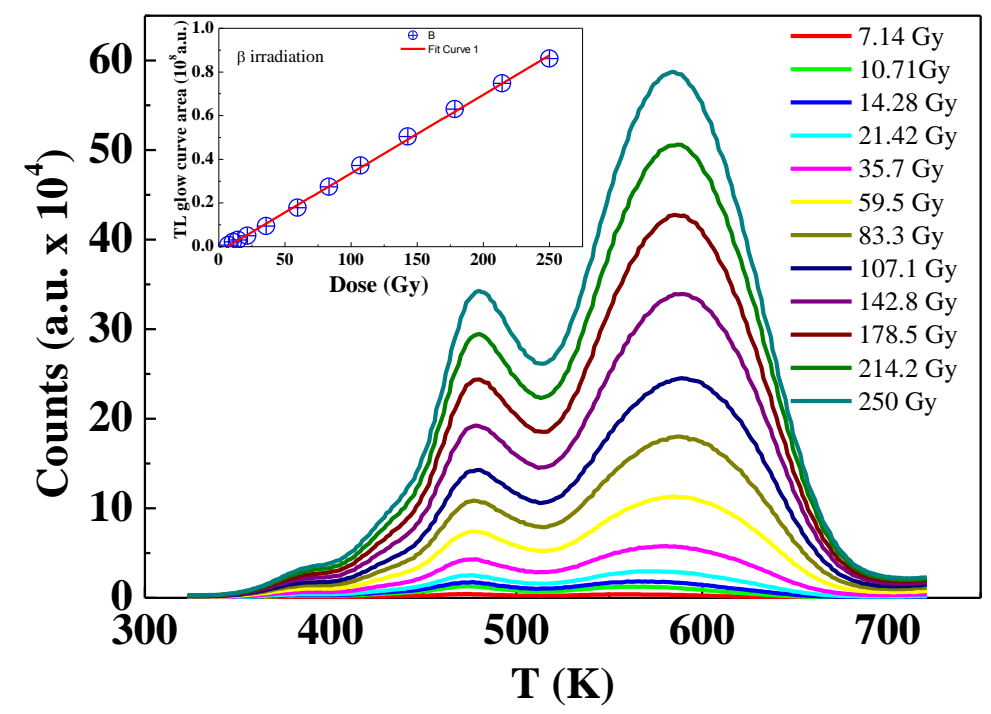

245 Figure 5. Thermoluminescence glow curves for different $\beta$ radiation exposure time (i.e. total dose). The alumina sample response is linear with the dose. (colour online)

Therefore, it has been possible to estimate the total plasma exposure dose from the ratio of the plasma

249 thermoluminescence glow curve area and the $\beta$ thermoluminescence glow curve area for a given radiation 250 dose (i.e. 10.7 Gy). To minimize the experimental error on the plasma radiation dose estimate the reference 251 signal obtained after exposure to the $\beta$ radiation source were collected on each sample after collecting the 252 plasma thermoluminescence glow curve. The experimental error can be due to small differences on the 253 geometrical area of the alumina slabs or to different GC intensities produced by $\beta$ irradiation due to 254 sensitization effects. The reference signal enable us also to verify that no radiation damage or sensitization 255 effect was produced on the dielectric surface.

256 The total plasma radiation dose for the different discharges are reported in Table 1. As can be observed, He 257 plasma is highly effective in filling several traps in the overall thermoluminescence temperature range while 258 the $\mathrm{O}_{2}$ plasma is the least effective one. It must be however pointed out that despite the not so much different 259 current from the case of $\mathrm{N}_{2}-\mathrm{O}_{2}$ discharge the number of counts observed in $\mathrm{O}_{2}$ plasma produced glow curve 260 is smaller. The counts reduction observed in $\mathrm{O}_{2}$ discharge can be ascribed to the presence of an higher level 261 of ozone producing de-trapping effect associated to the ozone-surface interaction. It has been in fact 262 demonstrated that ozone annealing efficiently eliminates the charge trapped in the dielectric used as 263 capacitor material [20]. 


\begin{tabular}{|c|c|c|c|c|c|c|c|c|c|}
\hline PLASMA & PeakN ${ }^{\circ}$ & $\begin{array}{l}T_{m} \\
(K)\end{array}$ & $\begin{array}{l}E_{t} \\
(e V)\end{array}$ & $\begin{array}{l}\text { Peak Area } \\
(\text { a.u.) }\end{array}$ & $\begin{array}{l}S \\
\left(s^{-1}\right)\end{array}$ & $\omega$ & $\begin{array}{l}\text { Dose } \\
(G y)\end{array}$ & $\begin{array}{l}E_{T o n} \\
(m J)\end{array}$ & $\begin{array}{l}Q_{c y c l e} \\
(C)\end{array}$ \\
\hline & 1 & 390 & 0.755 & 2310 & $1.6010^{9}$ & 27 & & & \\
\hline $\mathrm{O}_{2}$ & 2 & 458 & 0.888 & 20803 & $1.4610^{9}$ & 32 & & & \\
\hline FOM & 3 & 474 & 0.608 & 27687 & $4.6110^{5}$ & 48 & 1.15 & 8.9 & $1.410^{-9}$ \\
\hline \multirow[t]{3}{*}{$2.37 \%$} & 4 & 520 & 0.562 & 9353 & $3.7110^{4}$ & 61 & & & \\
\hline & 5 & 575 & 0.500 & 4064 & $2.1210^{3}$ & 83 & & & \\
\hline & 1 & 388 & 0.756 & 6550 & $3.7910^{9}$ & 27 & & & \\
\hline $\mathrm{N}_{2}-\mathrm{O}_{2}$ & 2 & 462 & 0.890 & 53984 & $2.4210^{9}$ & 32 & & & \\
\hline FOM & 3 & 473 & 0.620 & 118712 & $1.3210^{6}$ & 47 & 6.93 & 6 & $210^{-9}$ \\
\hline \multirow[t]{3}{*}{$1.5 \%$} & 4 & 530 & 0.562 & 36862 & $5.1210^{4}$ & 64 & & & \\
\hline & 5 & 571 & 0.500 & 188080 & $4.6210^{3}$ & 82 & & & \\
\hline & 1 & 388 & 0.852 & 11418 & $7.8410^{10}$ & 38 & & & \\
\hline \multirow{4}{*}{$\begin{array}{c}N_{2} \\
F O M \\
1.76 \%\end{array}$} & 2 & 418 & 0.99 & 12574 & $5.7210^{11}$ & 24 & & & \\
\hline & 3 & 475 & 0.759 & 290354 & $4.3610^{7}$ & 40 & 23.32 & 12 & $91^{-9}$ \\
\hline & 4 & 552 & 0.703 & 344381 & $6.7810^{5}$ & 57 & & & \\
\hline & 5 & 580 & 0.500 & 701399 & $3.7910^{3}$ & 84 & & & \\
\hline \multirow{5}{*}{$\begin{array}{c}\mathrm{He}-\mathrm{N}_{2} \\
\mathrm{FOM} \\
1.68 \%\end{array}$} & 1 & 390 & 0.868 & 12425 & $5.4010^{10}$ & 23 & & & \\
\hline & 2 & 422 & 1.090 & 15618 & $3.6310^{12}$ & 22 & & & \\
\hline & 3 & 475 & 0.770 & 296911 & $2.8710^{7}$ & 39 & 26.17 & 5.9 & $1.110^{-8}$ \\
\hline & 4 & 559 & 0.622 & 432134 & $4.7110^{4}$ & 64 & & & \\
\hline & 5 & 587 & 0.500 & 769263 & $1.6610^{3}$ & 86 & & & \\
\hline & 1 & 390 & 0.810 & 77232 & $1.7710^{10}$ & 25 & & & \\
\hline $\mathrm{He}$ & 2 & 425 & 0.991 & 216310 & $3.5010^{11}$ & 25 & & & \\
\hline$F O M$ & 3 & 470 & 0.758 & 1080551 & $5.4710^{7}$ & 38 & 69.37 & 7.55 & $2.810^{-8}$ \\
\hline \multirow[t]{2}{*}{$1.18 \%$} & 4 & 542 & 0.638 & 1235783 & $2.2010^{5}$ & 59 & & & \\
\hline & 5 & 576 & 0.500 & 1430699 & $4.1510^{3}$ & 83 & & & \\
\hline
\end{tabular}

265 Table 1. Best fit parameters extracted using the GlowFit program, i.e. Peak Temperature $\mathrm{T}_{\mathrm{m}}(\mathrm{K})$; Trap Energy $\mathrm{E}_{\mathrm{t}}(\mathrm{eV})$;

266 Peak Area, A (a.u.); Frequency factor, s ( $\left.\mathrm{s}^{-1}\right)$; Figure of Merit (FOM). The peak width $\omega$ and Dose (Gy) are calculated

267 as described in the text. The fitted glow curves refers to 30 minutes plasma exposure for gas mixture plasmas leading to

268 different discharge regime. In the table are reported the corresponding plasma macroscopic parameters i.e.: Energy $\mathrm{T}_{\text {on }}$

269 (mJ); plasma current charge per cycle $\mathrm{Q}_{\text {cycle }}(\mathrm{C})$.

271 The peak deconvolution procedure [14] helps to better elucidate on the plasma radiation thermoluminescence

272 glow curve features and involved trap energies. In figure 6 the deconvolution for the thermoluminescence 
274 figure of merit (FOM) for each fit.
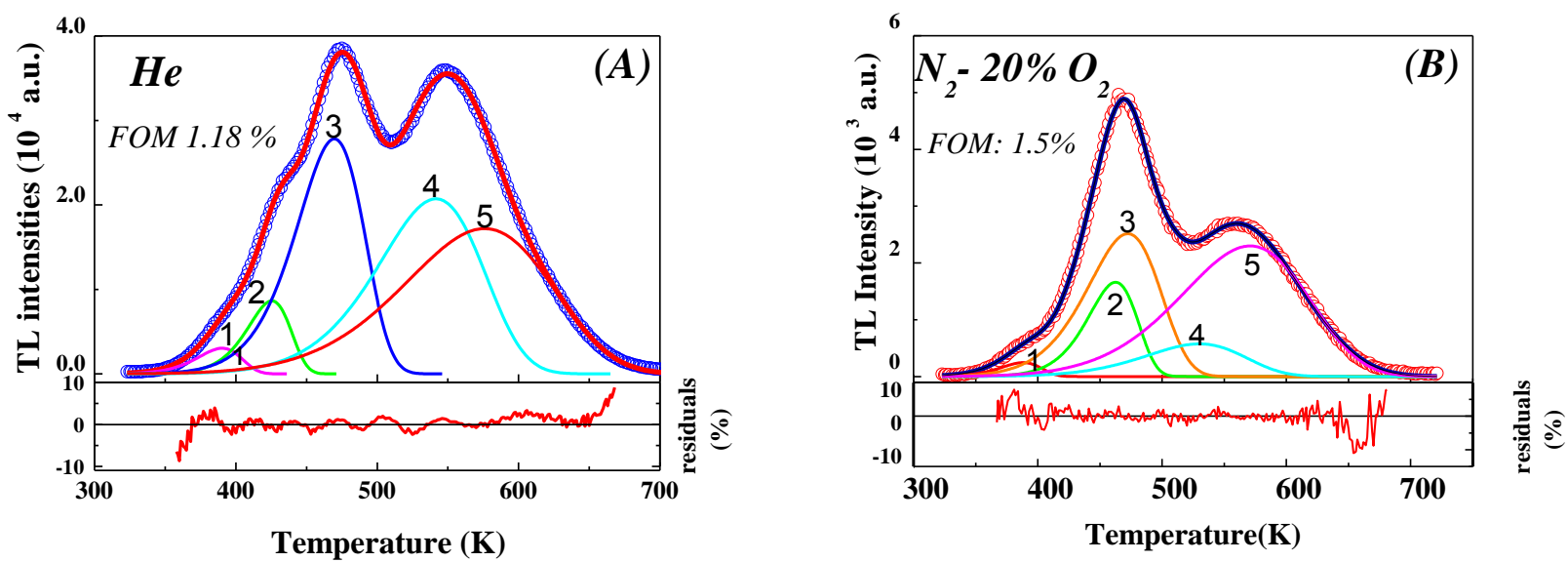

Figure 6. Glow curve fitting curve (red straight line) and deconvoluted peaks resulting as the output of the application of the GlowFit software on the experimental thermoluminescence curve (circles) for $\mathrm{He}$ (a) and $\mathrm{N}_{2} \mathrm{O}_{2}$ (synthetic air, (b)). The TL GC refers to 30 minutes plasma exposure. In the bottom part the residual values in the examined temperature range have been represented. The residual represents the difference at each temperature between the experimental and fitted points in the GC. The FOM values have been also reported. (colour online)

The best fit parameters obtained after peak deconvolution, referring to each peak, have been listed in Table 1 and represents the thermoluminescence glow peak maximum temperature $\left(T_{m}\right)$, trap activation energies $\left(\mathrm{E}_{\mathrm{t}}\right)$, frequency factor $S$, glow peak area (A) and figure of merit values (FOM)[21]. The frequency factor $s$ and has been found higher for the lowest temperature peaks. The FOM accounts for the goodness of thermoluminescence glow curve modeling and respect to previously published results [4], the fitting procedure has been improved by reducing the figure of merit (FOM) values less than 2\% (see Table 1).
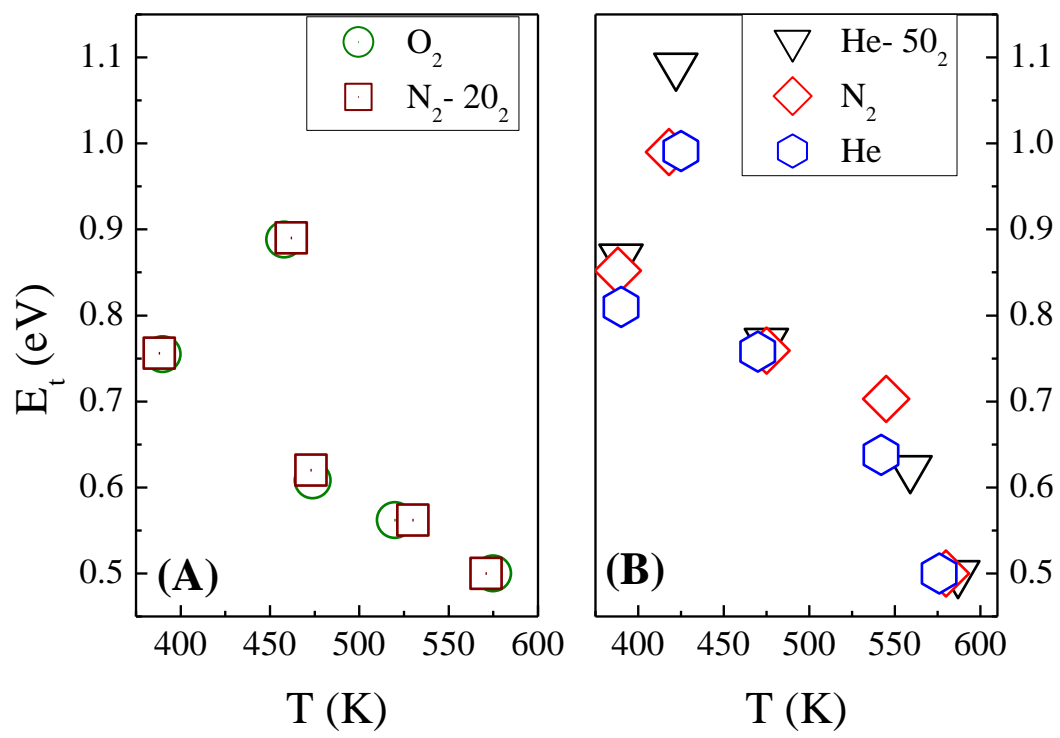

Figure 7. Correlation between the discharge regimes and the deconvoluted peak parameters $\left(T_{M}\right.$ and $\left.E_{t}\right)$ of Table 1: filamentary regimes (A) and glow and pseudo glow regimes (B). (colour online) 
292 The deconvolution of glow curve produced after plasma exposures evidence five contributing peaks. The results reported in Table 1 can be more easily understood if we consider figure 7.

It can be observed that traps filled by the plasma are similar in the case of air and oxygen discharge, but with a trapping efficiency higher for the air like plasma (see Table 1). We speculate that in $\mathrm{N}_{2}-\mathrm{O}_{2}$ plasma the lower ozone production leads to a lower ozone-mediated detrapping effect.

The glow curve intensities produced in the case of glow $(\mathrm{He})$ and pseudo glow $\left(\mathrm{N}_{2}, \mathrm{He}-\mathrm{N}_{2}\right)$ regime show very strong similarities. In particular the higher temperature peaks are the same with slight differences in the peak temperatures $(<15 \mathrm{~K})$ and energies $(<10 \mathrm{meV})$. These attributions are furthermore justified when considering the similar order of magnitude of the frequency factor $S$ values.

We can conclude that the different discharges characterized by different regimes (i.e. different plasma current and electron energy) are more or less effective in filling the electron traps in the dielectric materials. We can also speculate that the trap filled by different plasmas are keeping memory of the active species (metastable, or electronegative gases, etc.): in fact different trap at dielectric surface are filled giving a different signature on the thermoluminescence glow curve.

\subsection{Effect of the exposure time}

The thermoluminescence glow curves were collected for different total exposure time of the alumina samples

to $\mathrm{He}$ and $\mathrm{N}_{2}-\mathrm{O}_{2}$ plasmas, in order to observe the effect of two extreme plasma regimes (glow and filamentary respectively) on charge trapping. In figure 8 the obtained glow curves are reported together with the estimated dose for the different exposure time.
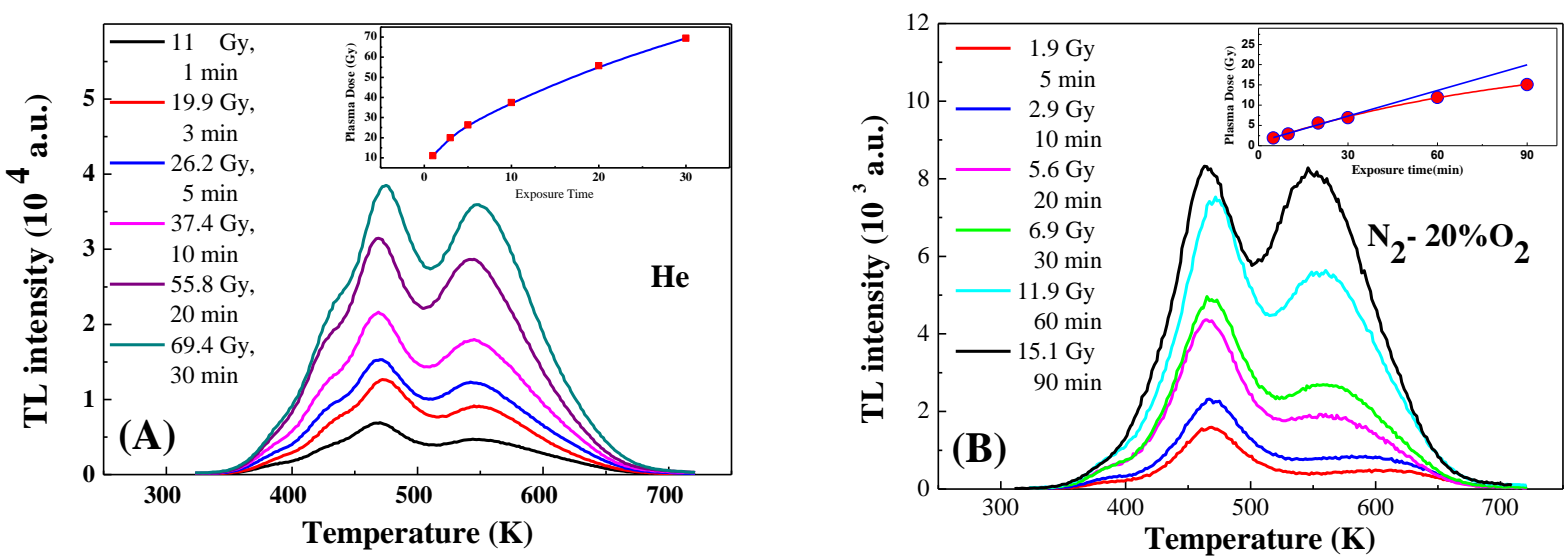

Figure 8. Thermoluminescence glow curves for different time exposure in the case of (a) Helium and air (b) discharge. In the legend the estimated total dose are reported together with the total exposure time. (colour online)

At the lowest doses, the thermoluminescence glow curve intensity is higher in the lower temperature region than in the high temperature region. Increasing the exposure time the thermoluminescence glow curve intensities for the two temperature region seem to converge to an almost common value. This effect is due to the presence of several trapping states that can act one as active and other as competitor traps. Generally, the 
trapping probability of the competitor trap is higher than that of the active one so that the glow peak area

321 tends to saturate due to the fast filling of competitor trap levels; after competitor level saturation more 322 electrons are trapped to the active states that shows a fast linear trap filling [15]. These effects may account for the behavior observed at the lowest doses in He and air discharge.

In the inset of figure 8 are represented the total thermoluminescence glow curve area values as a function of irradiation time, that in the case of beta irradiation can be easily converted in the total radiation dose. A saturation effect is clearly observed in $\mathrm{He}$ while in $\mathrm{N}_{2}-\mathrm{O}_{2}$ it is less marked. It has to be stressed that saturation effect has not been observed after $\beta$ irradiation both for doses similar or much higher than plasma ones.
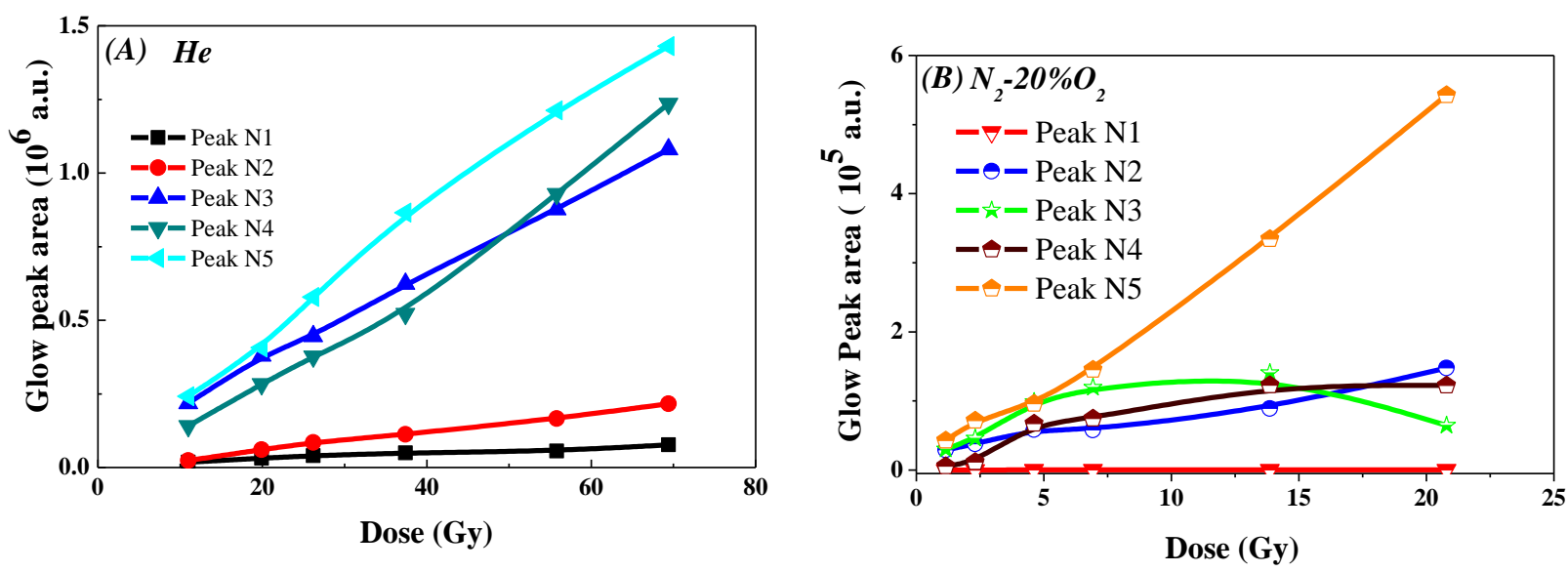

Figure 9. Thermo luminescence deconvoluted glow curve peak areas versus plasma dose for the case of $\mathrm{He}$ (a) and Air like plasma (b) discharge. N.i. represent the peak number as reported in Table 1. (colour online)

The dynamic behavior of thermoluminescence glow curve has been exploited by examining the evolution of each glow peak when changing the plasma doses. It has to be underlined that since plasma dose depends on the discharge regime, the examined dose range is different although exposure time is the same. Figure 9 shows the glow peak areas as a function of the corresponding irradiation dose under glow (He figure 9(a)) and filamentary $\left(\mathrm{N}_{2}-\mathrm{O}_{2}\right.$ (figure $\left.9(\mathrm{~b})\right)$ discharge regime. In the glow regime, the lower temperature and the highest temperature peak areas saturates(competitor traps), while intermediate temperature peak display a general linear behavior (active trap). In the filamentary regime, the highest temperature peak display an almost linear behavior (active trap) while the remaining peaks saturate with the dose (competitor trap).

The overall saturation effect observed in the case of plasma induced charge trapping can be explained if we consider that the plasma electron energy is typically of the order of a few $\mathrm{eV}$, i.e., five orders of magnitude lower than the beta source, leading to an electron penetration depth of around tens of nanometer [22]. Therefore the plasma electrons are effective mostly on the first monolayers of alumina surface where they are readily stopped rather than on the overall bulk, which is instead fully crossed by beta radiation. This means that the real volume of the sample that is irradiate by plasma is smaller. If we assume that the traps are randomly distributed in the sample this means that the number of traps that can be filled by the plasma radiation is considerably lower, and as a consequence the saturation dose is also lower [23]. This is 
furthermore confirmed by comparing the glow curve area vs dose collected after $\beta$ and plasma irradiation in the same dose range (up to $50 \mathrm{~Gy}$ ) that was found linear for the former while saturates for the latter. The strong correlation between the estimated plasma radiation dose and the plasma current is evidenced in figure 10. Several experiment were run for different gas mixtures and regime in $\mathrm{He}, \mathrm{He} / \mathrm{N}_{2}, \mathrm{~N}_{2}, \mathrm{~N}_{2} / \mathrm{O}_{2}$, the current was constantly monitored and the thermoluminescence measurements performed soon after each run, together with the corresponding calibration measurements by irradiation with $\beta$ radiation.

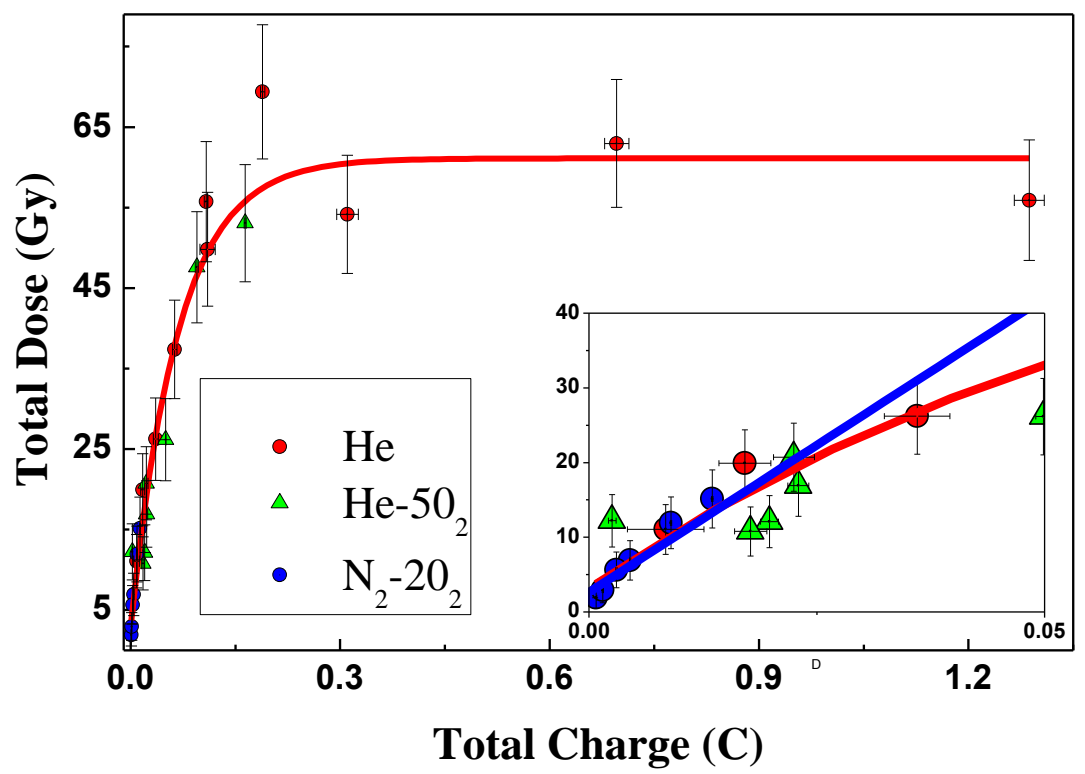

Figure 10. Estimated total doses for different plasmas as a function of the total discharge current estimated charge. The dose is linearly proportional to the charge up to dose of $15 \mathrm{~Gy}$ and deviates from linearity for higher doses, as observed when plotting the dose as a function of time exposure. (colour online)

For values of the total dose less or equal to $11 \mathrm{~Gy}$ the plasma radiation dose is linearly correlated to the total current transferred charge estimated by the integration of the current peak, while start to show a saturation effect for higher dose rate. In particular saturation is easily reached in the case of Helium, and $\mathrm{He} \mathrm{N}_{2}$ gas mixture. If we look carefully at figure 10 we can observe that if the estimated total plasma current transferred charge is the same, then estimated plasma radiation dose is also the same. This can be a further indication that the electrons hitting the surface are the main responsible for the charge trapping. Moreover the observed charge trapping generated by plasma exposure is a long lasting effect. A set of dedicated experiment was run in the case of He discharges in order to clearly show this effect.

Figure 11 is an example of the time evolution of the thermoluminescence curve collected on the same alumina sample just after the plasma exposure and by left the sample under dark for 70 hours after plasma exposure. The plasma irradiated sample was kept under dark in order to avoid the superposition of environmental light bleaching to fading effect. In the last case, the glow curve area is about a factor of two lower with respect to those of the as exposed sample evidencing the presence of the fading effect [15]. Here, the high temperature glow curve region relates to the initial one since the trap temperature and energy 
374 distribution were not modified. Conversely, changes in the temperature and energies of trapped charge 375 distribution (see Table 2) affect the low temperature glow curve region.

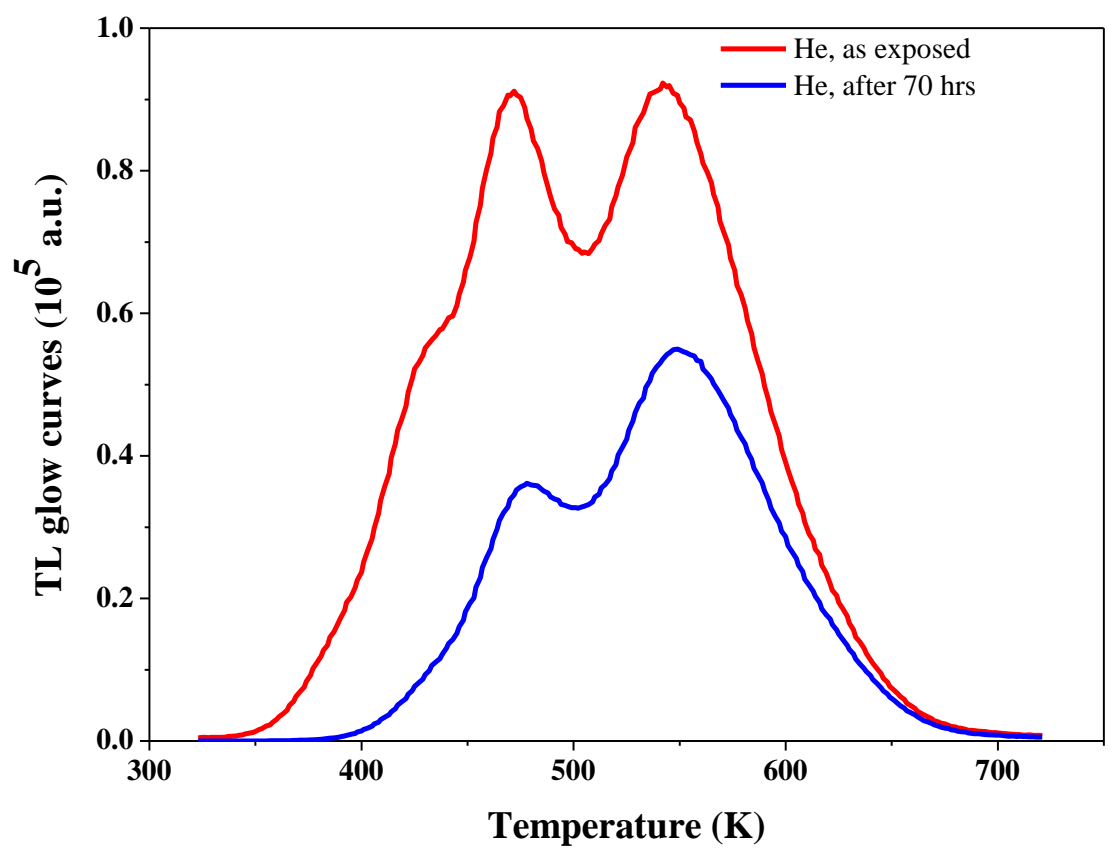

376

Figure 11. Thermoluminescence glow curve taken soon after the exposure to plasma and after 70 hours. (colour online)

The fading effect accounts for the high frequency factor $S$ of the lowest temperature peaks indicating that these traps are more easily released in time and can relax toward a more energetically stable trap distribution.

Table 2. Peak temperature and energies for glow curves taken just after exposure to plasma, 20, 40 and 70 hours.

\begin{tabular}{|c|c|c|c|c|}
\hline & $0 \mathrm{hrs}$ & 20 hrs & $40 \mathrm{hrs}$ & $70 \mathrm{hrs}$ \\
\hline \multicolumn{5}{|c|}{ Peak Temperatures (K) } \\
\hline $\mathrm{Pk}_{1}$ & 390 & & & \\
\hline $\mathrm{Pk}_{2}$ & 425 & 429 & 431 & 431 \\
\hline \multirow{2}{*}{$\mathrm{Pk}_{3}$} & 470 & 469 & 470 & 467 \\
\hline & & 494 & 494 & 487 \\
\hline $\mathrm{Pk}_{4}$ & 542 & 547 & 547 & 546 \\
\hline $\mathrm{Pk}_{5}$ & 576 & 574 & 576 & 574 \\
\hline \multicolumn{5}{|c|}{ Peak Energies $(\mathrm{eV})$} \\
\hline $\mathrm{Pk}_{1}$ & 0.810 & & & \\
\hline $\mathrm{Pk}_{2}$ & 0.990 & 1.18 & 1.31 & 1.38 \\
\hline \multirow{2}{*}{$\mathrm{Pk}_{3}$} & 0.760 & 1.03 & 1.04 & 1.14 \\
\hline & & 1.72 & 1.82 & 1.35 \\
\hline $\mathrm{Pk}_{4}$ & 0.640 & 0.77 & 0.78 & 0.76 \\
\hline $\mathrm{Pk}_{5}$ & 0.500 & 0.50 & 0.52 & 0.52 \\
\hline
\end{tabular}




\section{Conclusions}

387 Evidence of DBD plasma induced trap filling effect on alumina by using thermoluminescence techniques was clearly observed. The thermoluminescence signal originates from long living traps previously filled by plasma electron current, that are subsequently depleted by heating. The plasma generated thermoluminescence signal was found to be maximum for the Helium glow discharge plasma and in general higher for the homogenous discharge case. Evidence of a strong correlation between the thermoluminescence signal, i.e. the filled electron trap, and the total discharge current charge was demonstrated. Due to the low energy of the plasma electron the penetration depth of the electrons could only be few nanometers, leading to a saturation of the thermoluminescence signal with increasing exposure time. The generated charge traps was observed to last for several days, with a redistribution of the charge on deeper energy level. The redistribution of the trapped charge after several hours from plasma exposure tends to fill higher temperature trap with trap average energy slightly above $1 \mathrm{eV}$.

\section{Aknowledgments}

Authors wish to acknowledge Prof. A. Minafra for fruitful scientific discussion and Mr. G. Casamassima and Mr D. dell'Universita' degli Studi di Bari.

\section{References}

[1] M.Li, X.Wang, C.Li,H.Zhan and J.Xu, 2008, Appl.Phys.Lett 92, 031503

[2] J Ráhel' and D M Sherman, 2005, J. Phys. D: Appl. Phys. 38, 547

[3] Y. B. Golubovskii, V. Maiorov, and J. F. Behnke, 2002, J. Phys. D, 35, 751

[4] P. F. Ambrico, M. Ambrico, L. Schiavulli, T. Ligonzo, and V. Augelli, 2009, Appl. Phys. Lett. 94, 051501

[5] P. F. Ambrico, M. Ambrico, M. Šimek, A. Colaianni, G. Dilecce, and S. De Benedictis, 2009, Appl. Phys. Lett. 94, 231501

[6] A Meiners, M Leck and B Abel, 2009 , Plasma Sources Sci. Technol., 18045015

[7] H Luo, Z Liang, X Wang, Z Guan and, L Wang , 2010, J. Phys. D: Appl. Phys. 43155201

[8] G Dilecce, P F Ambrico and S De Benedictis, 2007, Plasma Sources Sci. Technol. 16511

[9] F Massines, N Gherardi, N Naudé and P Ségur, 2005, Plasma Phys. Control. Fusion 47 B577

[10] I. Radu, R. Bartnikas, M. R. Wertheimer, 2004, J. Appl. Phys., 95, 5994

[11] S. Celestin, G Canes-Boussard, O guaitella, A Bourdon and A Rousseau, 2008, J. Phys. D: Appl. Phys., 41205214

[12] http://www.risoe.dk/business_relations/Products_Services/Dosimetri/NUK_instruments/TL_OSL_readers.aspx

[13] M. Duran, F. Massines, G. Teyssedre, C. Laurent, Surf. Coat. Tech., 2001, 142-144, 743

[14] M. Puchalska, P. Bilski, 2006, Rad. Meas. 41, 659

[15] S.W.S. McKeever, Thermoluminescence of solids, Cambridge University Press, Cambridge, 1985

[16] Chen R and McKeever S WW S, Theory of Thermoluminescence and Related Phenomena, World Scientific Publishing, Singapore, 1997

[17] Horowitz, Y. S. and Yossian, 1995, D., Radiat. Prot. Dosim. 60 (1): 3.

[18] G. Baldacchini, P. Chiacchiaretta, V. Gupta, V. Kalinov, A. P. Voitovich, 2008, Phys. Solid State, 50, 1747

[19] H.G.Balian and N.W.Eddy, 1977, Nucl.Instrum.Methods 145389

[20] H. Kato, K. Soo Seol, M. Fujimaki, T. Toyoda, Y. Ohki and M. Takiyama, 1999, Jpn. J. Appl. Phys. 386791

[21] H.G.Balian and N.W.Eddy, 1977, Nucl.Instrum.Methods, 145389

[22] J. C. Ashley and V. E. Anderson, 1981, J. Electron Spectrosc. Relat. Phenom.24, 127

[23] J. Faï̇n, S. Sanzelle, Th. Pilleyre, D. Miallier, M. Montret, 1999, Rad. Meas. 30, 487 